\title{
Interaction of Tungsten tips with Laguerre-Gaussian beams
}

\author{
Abhisek Sinha ${ }^{1, *}$, Debobrata Rajak $^{2}$, Shilpa Rani $^{1}$, Ram $_{\text {Gopal }}{ }^{2}$, and Vandana Sharma ${ }^{1, * *}$ \\ ${ }^{1}$ Indian Institute of Technology Hyderabad, India \\ ${ }^{2}$ Tata Institute of Fundamental Research Hyderabad, India
}

\begin{abstract}
Interaction of femtosecond laser pulses with metallic tips have been studied extensively and they have proved to be a very good source of ultrashort electron pulses. We present our study of interaction of Laguerre-Gaussian (LG) laser modes with Tungsten tips. We report a change in the order of the interaction for LG beams and the difference in the order of interaction is attributed to ponderomotive shifts in the energy levels corresponding to the enhanced near field intensity supported by numerical simulations.
\end{abstract}

\section{Introduction}

Laguerre-Gaussian (LG) modes are a solution to the paraaxial Helmholtz equation with a cylindrical symmetry. The most interesting properties of LG beams are a result of their topological phase structure. The phase of an LG beam continually increases counter-clockwise, on a crosssection of the beam, along a closed loop from 0 to $2 \pi l$, where $l$ is an integer called the topological charge. Since the angle 0 is equal to $2 \pi l$, a continuous distribution of phase is obtained resembling the topological structure of a mobius strip [1]. A phase singularity, also called a vortex is formed at the centre of the beam where the phase remains undefined. Due to this azimuthal phase variation, the wavefront appears twisted in shape. This twisting of the beam is identified as the OAM of the beam and the pitch of the twist determines the magnitude of the OAM which is related to the topological charge. It was shown that the projection of the OAM along the direction of propagation is equal to $i \hbar$ per photon averaged over the beam [2]. Thus, individual photons carry orbital angular momentum in addition to spin angular momentum in LG modes.

In practice, such beams are obtained from Gaussian modes using computer generated holograms $[3,4]$ or spiral phase plates $[5,6]$. Other methods involve astigmatic mode conversions where high order Hermite-Gaussian (HG) beams are passed through a pair of cylindrical lenses and the Gouy phase shift thus introduced results in the conversion of the HG mode to an LG mode [7]. Lower order LG modes were generated in the cavity of solid state lasers using nanoscale mode selection elements [8]. The detection and characterization of the mode of the resultant beam can be done using interferometric techniques $[9,10]$ or by studying diffraction of the beam through different apertures $[11,12]$.

\footnotetext{
*e-mail: ph18resch01002@iith.ac.in

**e-mail: vsharma@phy.iith.ac.in
}

LG beams are central to various applications in diverse fields. In the field of communication, LG beams were used to encode multiple bits in a single photon [13] with an inherent security feature that does not require any mathematical or quantum mechanical encryption. LG beams are used in imaging applications to achieve super-resolution upto the order of $\lambda / 25$ in Stimulated Emission Depletion (STED) microscopy [14, 15]. LG modes are well suited for Gravitational wave detectors as they are reported to reduce the influence of thermal noise in such systems $[16,17]$. Other applications include micromachining [18] and optical tweezing [19].

Considerable amount of research has been done on the properties of LG modes and the transfer of OAM to matter at various scales. For instance, it was shown in the case of photoexcitation and photoionization of atoms by OAM beams, the selections rules can be different than those of plane waves due to transfer of OAM [20]. Properties of above threshold ionization spectra by OAM beams were calculated and predicted the existence of photoelectrons emitted in the direction of laser propagation [21]. In high harmonic generation, it was shown that the OAM was conserved in the process of generation of higher harmonics of the fundamental beam [22]. In metal nanoparticles, transfer of OAM to surface plasmons have been theoretically demonstrated and the excited plasmon mode was shown to be determined by the Total Angular Momentum (TAM) transferred in the excitation process [23]. For larger particles, the mechanical equivalence of spin and orbital angular momentum was established by the observation of cancellation (or addition) of spin angular momentum by orbital angular momentum to give total angular momentum [24].

In this report, we have presented our study of interaction of femtosecond LG beams with metallic tips. The electron emission and their dynamics from metallic tips illuminated by femtosecond laser pulses are well known $[25,26]$. When light is focused on metallic tips of sizes 
smaller than the wavelength of the incident light, the field value near the apex of the tip rises several times the incident field [27]. This enhanced near field leads to emission of photoelectrons from the tip surface. In the case of vortex beams, this near field enhancement can be different and may lead to different emission properties. A comparison of the electron emission rates is presented and we show a change in the electron emission property for LG modes.

\section{Experimental Methods}

A schematic representation of the experimental setup is shown in figure (1). A Ti-Sapphire laser lasing $25 \mathrm{fs}$, $800 \mathrm{~nm}$ pulses at a repetition rate of $1 \mathrm{kHz}$ was used as the source in the experiment. A combination of halfwaveplate and polarizer was used to control the intensity of the laser beam. A spiral phase plate was used to obtain the desired LG mode of topological charge, $l=1$. To detect the emitted electrons, a channeltron detector was placed parallel to the tip axis. The output signal from the detector was then fed to a Digital Oscillosocope triggered by a high speed photodiode. The position of the tip with respect to the laser beam was constantly monitored using a CCD camera.

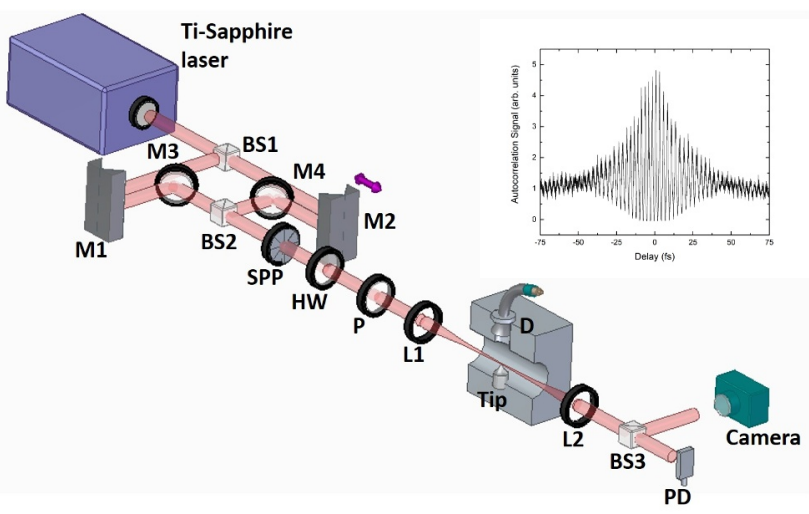

Figure 1: Schematic of the experimental setup used in the experiment. BS1, BS2, BS3: Beam Splitters; M1, M2: Movable mirrors; M3, M4: Mirrors; SPP: Spiral Phase Plate; HW: Half Waveplate; P: Polarizer; L1, L2: Focusing Lens; D: Channeltron Detector; PD: Photo diode. Inset: Interferometric Autocorrelation Trace (IAT) with the Tungsten tip as the nonlinear element.

\section{Results}

Inset in figure (1) shows the Interferometric Autocorrelation Trace (IAT) with the tip used as the nonlinear element. The emission process was fast enough for the measurement of $35 \mathrm{fs}$ pulses ruling out thermal emission with characteristic timescales of 100 fs to 1 ps [28, 29]. Figure (2) shows the electron emission rates as a function of the average power for Gaussian beams. The data depicted in a log-log plot forms a straight line and the value of the slopes are 2 and 1.6 respectively for tip voltages $0 \mathrm{~V}$ and $40 \mathrm{~V}$. For the case of an LG beam, figure (3), containing
OAM $(l=1)$, the slopes of the log-log plot are nearly equal to 1 for both $0 \mathrm{~V}$ and $40 \mathrm{~V}$.

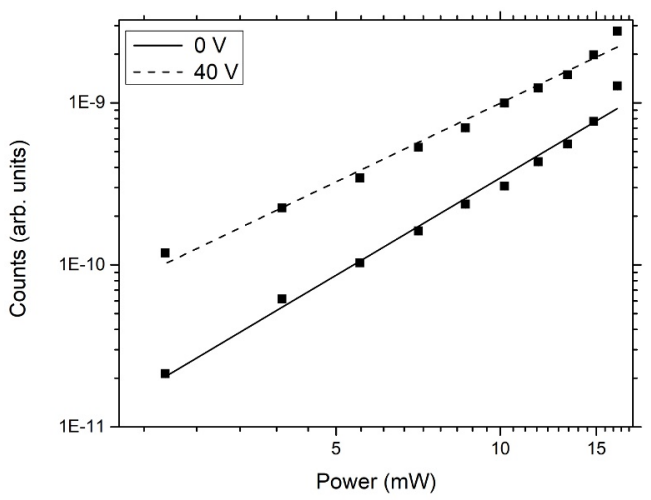

Figure 2: Gaussian beam when focused on the tip. In a $\log$-log plot, the slope of the curve represents the exponentiating index $\left(\Gamma=\alpha_{n} I^{n}\right)$. The values of $n$ are 2 (solid) and 1.6 (dash). $\Gamma$ is the emission rate, $I$ the intensity of the radiation, $n$ the number of photons absorbed and $\alpha_{n}$ the cross-section of the interaction.

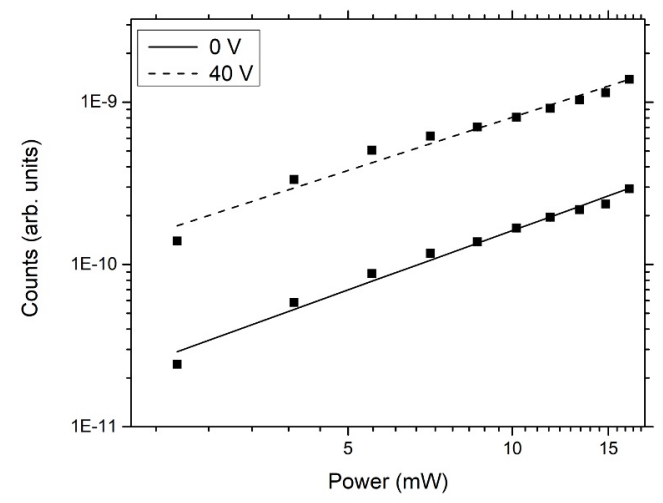

Figure 3: Counts variation with average laser power for OAM beams. Slope of the lines are close to 1 for both of the tip voltages.

\section{Discussions}

It is seen that in both Gaussian and LG beams, the yield of $40 \mathrm{~V}$ is higher than $0 \mathrm{~V}$. This is because applying a potential on the tip decreases the height of the potential barrier giving rise to a higher ionization probability. The slope of the plot represents the number of photons absorbed in the process of ionization. As seen in numerical simulations, the intensity distribution indicates a higher field value at the tip apex for Gaussian beams. The ponderomotive energy due to this field distribution on the apex of the tip shifts the continuum [30] higher for Gaussian beams than LG beams. As a result of this shift in the continuum, the order 
of the interaction for the Gaussian beam is higher than that of the LG beam.

\section{Conclusion}

In this work we have explored the interaction of metallic tips with LG laser modes. The order of interaction for Gaussian beams was seen to be higher than LG beams. It is a result of a larger field enhancement factor for Gaussian beams near the apex of the tip that lifts the continuum higher so that more photons are required for ionization.

\section{References}

[1] Y. Shen, X. Wang, Z. Xie, C. Min, X. Fu, Q. Liu, M. Gong, X. Yuan, Light: Science \& Applications 8, 1 (2019)

[2] L. Allen, M.W. Beijersbergen, R. Spreeuw, J. Woerdman, Physical review A 45, 8185 (1992)

[3] N. Heckenberg, R. McDuff, C. Smith, A. White, Optics letters 17, 221 (1992)

[4] J. Arlt, K. Dholakia, L. Allen, M. Padgett, Journal of modern optics 45, 1231 (1998)

[5] K. Sueda, G. Miyaji, N. Miyanaga, M. Nakatsuka, Optics express 12, 3548 (2004)

[6] M. Beijersbergen, R. Coerwinkel, M. Kristensen, J. Woerdman, Optics communications 112, 321 (1994)

[7] M.W. Beijersbergen, L. Allen, H. Van der Veen, J. Woerdman, Optics Communications 96, 123 (1993)

[8] D. Lin, J. Daniel, W. Clarkson, Optics letters 39, 3903 (2014)

[9] M. Harris, C. Hill, P. Tapster, J. Vaughan, Physical Review A 49, 3119 (1994)

[10] M. Padgett, J. Arlt, N. Simpson, L. Allen, American Journal of Physics 64, 77 (1996)

[11] J.P.C. Narag, N. Hermosa, Physical Review Applied 11, 054025 (2019)

[12] L.E. de Araujo, M.E. Anderson, Optics letters 36, 787 (2011)

[13] G. Gibson, J. Courtial, M.J. Padgett, M. Vasnetsov, V. Pas'ko, S.M. Barnett, S. Franke-Arnold, Optics express 12, 5448 (2004)
[14] K.I. Willig, S.O. Rizzoli, V. Westphal, R. Jahn, S.W. Hell, Nature 440, 935 (2006)

[15] S.W. Hell, J. Wichmann, Optics letters 19, 780 (1994)

[16] M. Granata, C. Buy, R. Ward, M. Barsuglia, Physical review letters 105, 231102 (2010)

[17] A. Allocca, A. Gatto, M. Tacca, R. Day, M. Barsuglia, G. Pillant, C. Buy, G. Vajente, Physical Review D 92, 102002 (2015)

[18] K. Ladavac, D.G. Grier, Optics express 12, 1144 (2004)

[19] H. He, M. Friese, N. Heckenberg, H. RubinszteinDunlop, Physical review letters 75, 826 (1995)

[20] A. Picón, J. Mompart, J.V. de Aldana, L. Plaja, G. Calvo, L. Roso, Optics express 18, 3660 (2010)

[21] B. Böning, W. Paufler, S. Fritzsche, Physical Review A 98, 023407 (2018)

[22] G. Gariepy, J. Leach, K.T. Kim, T.J. Hammond, E. Frumker, R.W. Boyd, P.B. Corkum, Physical review letters 113, 153901 (2014)

[23] K. Sakai, K. Nomura, T. Yamamoto, K. Sasaki, Scientific reports 5, 1 (2015)

[24] N. Simpson, K. Dholakia, L. Allen, M. Padgett, Optics letters 22, 52 (1997)

[25] M. Krüger, C. Lemell, G. Wachter, J. Burgdörfer, P. Hommelhoff, Journal of Physics B: Atomic, Molecular and Optical Physics 51, 172001 (2018)

[26] M.F. Ciappina, J.A. Pérez-Hernández, A.S. Landsman, W.A. Okell, S. Zherebtsov, B. Förg, J. Schötz, L. Seiffert, T. Fennel, T. Shaaran et al., Reports on Progress in Physics 80, 054401 (2017)

[27] Y.C. Martin, H.F. Hamann, H.K. Wickramasinghe, Journal of applied physics 89, 5774 (2001)

[28] P. Hommelhoff, C. Kealhofer, M.A. Kasevich, Physical review letters 97, 247402 (2006)

[29] B. Barwick, C. Corder, J. Strohaber, N. ChandlerSmith, C. Uiterwaal, H. Batelaan, New Journal of Physics 9, 142 (2007)

[30] M. Schenk, M. Krüger, P. Hommelhoff, Physical review letters 105, 257601 (2010) 